\title{
Role of the nervous system in cancers: a review
}

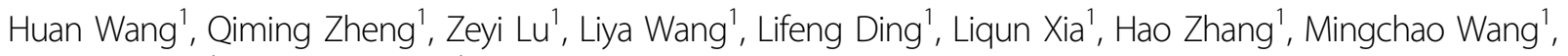 \\ Yicheng Chen ${ }^{1}$ and Gonghui Li ${ }^{1}$
}

\begin{abstract}
Nerves are important pathological elements of the microenvironment of tumors, including those in pancreatic, colon and rectal, prostate, head and neck, and breast cancers. Recent studies have associated perineural invasion with tumor progression and poor outcomes. In turn, tumors drive the reprogramming of neurons to recruit new nerve fibers. Therefore, the crosstalk between nerves and tumors is the hot topic and trend in current cancer investigations. Herein, we reviewed recent studies presenting direct supporting evidences for a better understanding of nerve-tumor interactions.
\end{abstract}

\section{Facts}

1. Nerves, as components of the tumor microenvironment, are associated with cancer outcomes.

2. Nerve transmitters and neurotrophic factors play an essential role in tumor progression.

3. Perineural invasion is a common characteristic of some tumors.

\section{Open questions}

1. How do tumors and nerves have crosstalk?

2. What is the molecular mechanism underlying of perineural invasion?

3. How can we appropriately target the nerves to prevent the tumor progression?

\section{Introduction}

The tumor microenvironment (TME) is closely related to tumor initiation, progression, and metastasis. It consists of the extracellular matrix, fibroblasts, adipose cells, immune-inflammatory cells, blood, and lymphatic vascular networks ${ }^{1}$. The functions of oncogenes and tumor suppressor genes in tumorigenesis have long been

\footnotetext{
Correspondence: Yicheng Chen (chenyicheng@zju.edu.cn) or Gonghui Li (3193119@zju.edu.cn)

'Department of Urology, Sir Run Run Shaw Hospital, Zhejiang University School of Medicine, Hangzhou, Zhejiang 310016, China

Edited by Joern Steinert
}

identified. In recent years, the concept of cancer biology has shifted from studying the genetics of tumor cells alone to the field of complicated interplay between tumor cells and the TME. The elements of this interplay, especially tumor angiogenesis, have been wellcharacterized in previous research ${ }^{2}$. Hence, nerves as components of the TME have been increasingly proved to regulate aberrant tissue function, including cancer progression. The crosstalk between nerves and cancer cells has been well-established for a variety of cancers, including pancreatic, prostate, breast, head and neck cancers, as well as cholangiocarcinoma ${ }^{3-7}$. This association is often correlated with poor outcomes. Upon the recognition of the significance of nerve-cancer interactions, the National Cancer Institute has convened their first meeting to explore the "Role of Nerves in Cancer progression" in March $2015^{8}$.

Many research groups have established various models and demonstrated their own hypothesis to answer the main relevant questions, such as the influence of neuroactive molecules on cancers, the contribution of different nerves to cancers, or how cancers and nerves communicated. Technological advances in precise nerve imaging and manipulation has also allowed some progress in understanding the molecular mechanisms behind the crosstalk between cancers and nerves.

In this review, current theories on the communication channels and the functional relationship between cancers and nerves are summarized. The cellular and molecular 
mechanisms of the process based on recent studies are also reviewed. Thus, a more comprehensive understanding of the interplay between tumor cells and nerves may be useful to devise new strategies for cancer therapy.

\section{Nerves create a unique TME and associate with the outcome}

Nerves, consisting of a variety of cells such as neurons and neuroglia, form a unique type of TME. Nerves and related nerve cell markers have been detected in various tumors, including those of the head and neck, prostate, breast, cervix, esophagus, stomach, colorectum, and pancreas $^{9-15}$. When cancers develop from preneoplastic lesions to obvious cancer, nerve density nearly doubles compared with the non-neoplastic tissue ${ }^{16}$. More importantly, nerves found in the tumor increase the malignancy and are often correlated with poor outcomes. Huang et al. ${ }^{10}$ confirmed that nerve fibers in breast cancer were significantly correlated with poor differentiation, lymph node metastasis, high clinical staging, and the triplenegative subtype. In the same manner, nerve fiber density was also correlated with tumor size, margin status, lymph node metastasis, pathological tumor, and American Joint Committee on Cancer stages, as well as survival time in pancreatic cancers ${ }^{7,15}$. A further study also found nerves to be involved in angiogenesis related to tumor growth ${ }^{17}$. Horn et al. ${ }^{18}$ associated nerves with the recurrence of rectal adenocarcinoma after the radical surgery. All the above evidences support that nerves play crucial roles in tumor progression.

Tumor-nerve interactions are characterized by two aspects as follows: (i) tumor cells can secrete neurotrophic factors, neurotransmitters, and axon guidance molecules via paracrine signaling to drive neuron reprogramming, and thereby recruit the nerves or invade the existing nerves; (ii) nerves can also secrete neuroactive molecules to interact with the receptors of tumors or the TME for the tumor cells to proliferate, invade, and metastasize. These two aspects will be discussed in detail below.

\section{Effect of tumor cells on nerves}

\section{Perineural invasion in cancers}

Cancer cells infiltrate inside or around nerves in the process of perineural invasion (PNI), which can be observed before lymphatic or vascular invasion ${ }^{19,20}$. The PNI process, first characterized in head and neck carcinoma in 1856 by Batsakis ${ }^{19}$, has been described in detail by several reviews ${ }^{20-24}$. The first definition, however, was unclear and PNI attracted little attention in subsequent research. In 2009, Liebig et al. ${ }^{20}$ summed up previous studies and characterized PNI by the close proximity of tumor to nerve, and as a process involving at least $33 \%$ of its circumference or tumor cells present within any of the three layers of the nerve sheath. Thus, PNI is more commonly detected in aggressive cancers. The incidence of PNI was reported as up to $80 \%$ in head and neck cancers, $75 \%$ in prostate cancers, $98 \%$ in pancreatic cancers, $33 \%$ in colorectal cancers ${ }^{20}$, and $75 \%$ in cholangiocarcinoma ${ }^{25}$. Evidences indicating that PNI is a significant predictor of overall survival or disease-free survival of tumor patients is emerging ${ }^{9,26-30}$.

PNI involving structural nerve damage directly leads to cancer-associated pain ${ }^{21}$. More importantly, it is considered a potential pathway for cancer cells dissemination and metastasis in the same way as vascular and lymphatic channels ${ }^{31}$. Saloman et $\mathrm{al}^{3}$ showed that tumor cells invaded neural tissue before the onset of tumorigenesis. In addition, at the early stage of cancer, pancreas acinarderived cells were found to migrate along sensory neurons into the spinal cord, providing evidence that PNI could be a potential route of metastasis ${ }^{3}$. Recent studies suggest that the neural tracking hypothesis likely explains the mechanisms of PNI. Cancer cells track along or around a nerve after infiltrating into perineural space in the process of nerve injury, which in turn promotes neural regeneration. The damage of perineurium caused by invading cancer cells leads to a cascade of inflammation cytokines, thus forming a unique cellular and biochemical microenvironment around the nerve, named perineural niche ${ }^{32-35}$. This perineural niche includes various cellular components and may regulate the neural tracking to facilitate the $\mathrm{PNI}^{36,37}$ (Fig. 1).

In summary, PNI is a special means of tumor-nerve communication that is correlated to tumor progression. However, the understanding of PNI mechanisms remains limited, as an appropriate model to imitate the complex interactions between tumor and nerve fibers has not been proposed.

\section{Neoneurogenesis}

Neoneurogenesis (also called innervation) is the process when cancer cells react to nerve regeneration and recruit new axons into the tumor tissue, similar to angiogenesis. In contrast to the central nervous system cells, peripheral nerve cells have the ability to regenerate after injury ${ }^{38}$. Neoneurogenesis is a highly complex biological phenomenon, which remains to be elucidated. The neonerves originating from different nerves play different roles, or even opposite roles, in various tumors; the corresponding results are presented in Table 1.

The peripheral nerve system (PNS) includes the sympathetic and parasympathetic nerves, and maintains the homeostases of the body. The neurotransmitter of the sympathetic nerves is norepinephrine, whereas that of the parasympathetic nerves is acetylcholine (Ach), which both play important roles in the cellular communication. These coordinated systems control the blood pressure, $\mathrm{pH}$, thermoregulation, and metabolism, to adapt to external 


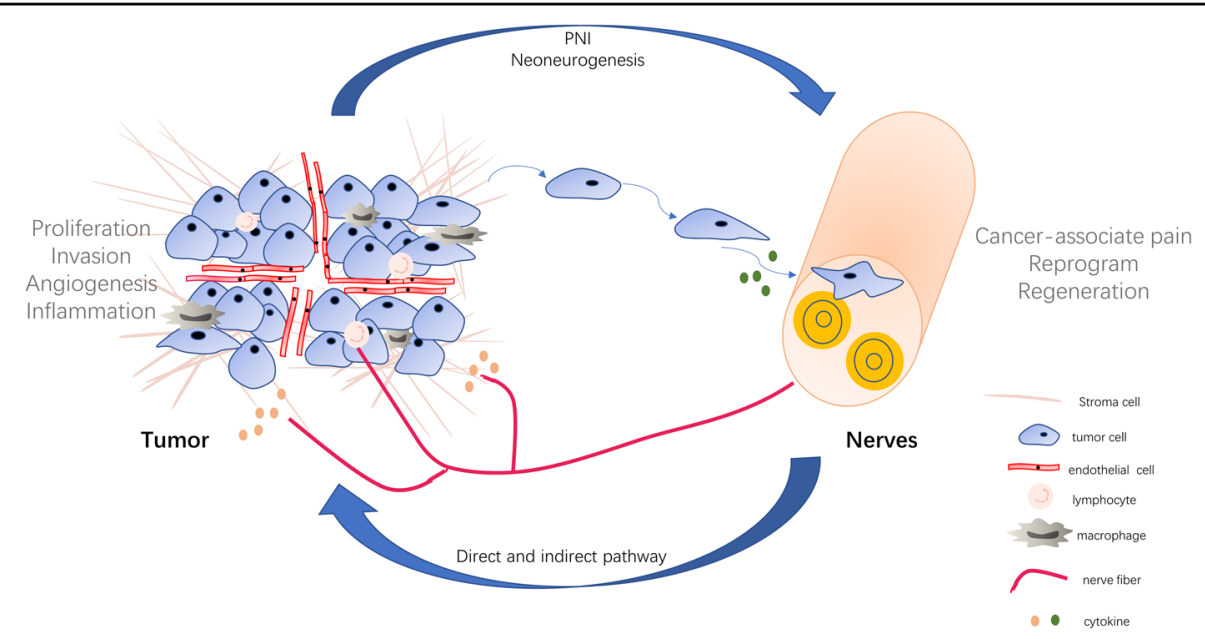

Fig. 1 The nerve-tumor crosstalk. Nerves can secrete the neuroactive molecules that act on tumor cells, lymphocytes, and macrophages to promote tumor proliferation, invasion, angiogenesis, and inflammation. In turn, tumor cells migrate to nerves and damage them to induce the cancer-associated pain. Moreover, tumor cells secrete cytokines, which drive nerve reprogramming and regeneration. PNI perineural invasion.

and internal pressures ${ }^{39}$. Sympathetic and parasympathetic nerves usually have opposing effects on a given tissue, such that they increase the activity of one system, whereas decrease the activity of the other. More specifically, sympathetic nervous activity increases the flow of blood rich in nutrients to tissues that need it during emergency "fightor-flight" reactions. Meanwhile, the parasympathetic system predominates during quiet, resting conditions. In summary, the PNS is affected by the tissues it innervates and responds to the changes in the microenvironment ${ }^{40}$. Therefore, certain lines of evidences have linked neoneurogenesis in a tumor to the PNS, including both sympathetic and parasympathetic nerves ${ }^{41}$. Prostate cancer neoneurogenesis was recognized relatively early ${ }^{42}$, whereas it was only later that Magnon et al. ${ }^{6}$ first demonstrated that the formation of autonomic nerve fibers in prostate cancer was required for cancer development and progression. In various mouse models, sympathetic nerve ablation, including that by both chemical or surgical method, could prevent prostate cancer development. Also, parasympathetic destruction could suppress the dissemination and invasion of prostate cancer. In a retrospective study, a high density of sympathetic and parasympathetic nerves was detected in tumors, and this was associated with poor clinical outcomes. Consistently with the results for prostate cancer, sympathectomy by the bilateral removal of superior cervical ganglia inhibited the tongue tumor growth and invasiveness ${ }^{43}$. Interestingly, breast cancer growth and progression were accelerated after sympathetic nerves stimulation, but were suppressed following the stimulation of parasympathetic nerves ${ }^{4,10,44}$. These findings suggested that the innervation of sympathetic and parasympathetic nerves play different roles in different cancers.
In addition to the parasympathetic and sympathetic nerves, sensory nerves also participated in the tumor progression. For example, sensory nerves may drive inflammation to accelerate precancerous lesions to pancreatic cancer via neurogenic mechanisms ${ }^{45,46}$. At the early stage of pancreatic cancer, the expressions of pancreatic neurotrophic factors change and sensory innervation obviously increases. At later stages, cells of pancreatic origin could migrate to the sensory ganglia and the spinal cord. The above findings prove that the sensory nerves participated in all stages of pancreatic cancers including tumorigenesis and progression ${ }^{47}$. Furthermore, the ablation of sensory neurons in a genetic model of pancreatic cancers showed a suppressive effect on tumor initiation and progression ${ }^{3}$. In a similar manner, sensory neurons were also shown to play a direct role in tumor formation in basal cell carcinoma ${ }^{48}$.

The vagus nerve, which contains both parasympathetic and sensory axons in a mixed nerve ${ }^{49}$, has been demonstrated to play completely inverse roles in pancreatic ductal adenocarcinoma and gastric cancer. Using three models of gastric cancer, Zhao et al. ${ }^{50}$ proved that vagotomy or pharmacological denervation of only the stomach portion decreased tumor progression and prolonged survival when performed in later cancer stages. Denervation particularly affected the renewal of the stem cell compartment of gastric tumors and was also able to enhance the effect of chemother$\mathrm{apy}^{50}$. In addition to the vagus nerve, enteric nerves are also involved in gastric cancer initiation and progres$\operatorname{sion}^{51,52}$. In contrast to the cancer-inducing effects in the gastric cancer, vagus nerves had an antitumor effect in pancreatic cancer ${ }^{53,54}$. Vagotomy accelerated pancreatic tumorigenesis and enhanced tumor growth 
Wang et al. Cell Death Discovery (2021)7:76

Page 4 of 12

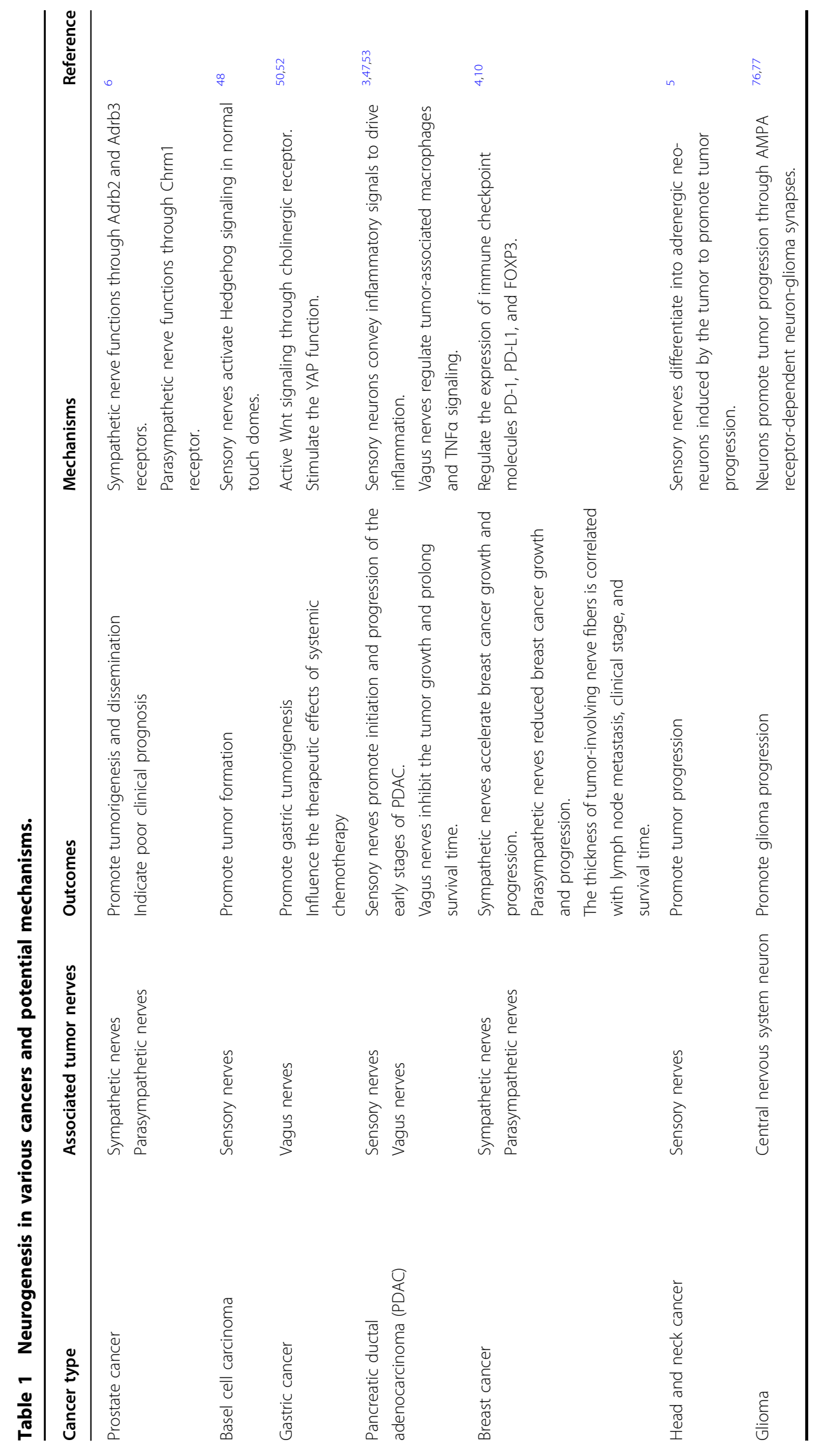

Official journal of the Cell Death Differentiation Association 
through recruiting tumor-associated macrophages (TAMs) and mediating the inflammation ${ }^{55,56}$.

According to novel findings, newly formed adrenergic nerve fibers in neck and head cancer originated from the sensory neurons and are not the infiltrations of preexisting adrenergic nerves ${ }^{5}$. Hence, signals that promoted tumor growth are also regulated by newly formed adrenergic nerve fiber instead of pre-existing adrenergic nerves. Furthermore, Amit et al. ${ }^{5}$ identified the adrenergic differentiation signature by comparing the transcriptomes of cancer-associated neurons with those of endogenous neurons. Their investigation of mechanisms revealed that TP53 loss in head and neck cancer drives sensory nerves reprogramming through the delivery of cancer-derived exosomes lacking miR-34a. Moreover, tumor growth was inhibited by sensory denervation or the pharmacological blockade of adrenergic receptors, but not by the chemical sympathectomy of pre-existing adrenergic nerves. These results indicated that cancer cells drive the neuron reprogramming to promote tumor progression. However, the potential role of neuron reprogramming induced by cancer cells in other tumors remains to be established.

Another study revealed that nerves emerging in tumors can also originate from the central nervous system ${ }^{57}$. In mouse models of prostate cancer, neural progenitors expressing doublecortin $(\mathrm{DCX}+)$ in the subventricular zone, egress into the circulation through disrupting the blood-brain barrier. These cells then infiltrate and reside in the tumor and can generate new neurons. Hence, the genetic depletion of DCX + cells inhibit the prostate cancer progression, whereas DCX + cells transplantation promotes prostate tumor growth and metastasis. These results provide new insights into the origin of tumor nerves, but the mechanisms of how solid tumors communicate with the central nervous system remain to be elucidated. In addition, the seeds of neoneurogenesis in tumors can also be mesenchymal stem cells (MSCs) derived from the bone marrow. Tumors recruited MSC, which in turn can differentiate into neurons under proper conditions in the $\mathrm{TME}^{58}$.

In summary, PNI and neoneurogenesis often occur and function together, thus providing the structural foundation for tumor-nerve communication.

\section{Direct effects of nerves on tumor}

Tumors affect nerve behavior but, more importantly, nerves also play important roles in regulating tumor progression through direct or indirect pathways. As cell membranes in tumors bear receptors that respond to neurotropic factors or neurotransmitters, the nerves release such molecules to promote tumor progression in a paracrine manner. Hence, innervations can release neurotransmitters directly into a synapse formed by neurons and tumor cells to transfer the excitatory signal. In the following section, we describe the two main direct effects of nerves on tumor progression.

\section{Paracrine mode}

Nerves, including neurons and Schwann cells, can modulate the biological behavior of cancer cells and influence tumor progression through the paracrine mode. From a broad perspective, neuroactive molecules released by the nerves involved in tumor-nerve interaction can be divided into three main families as follows: (i) neurotropic factors, such as nerve growth factor (NGF), brain-derived neurotrophic factor (BDNF), glial cell line-derived neurotrophic factor, and others; (ii) axon guidance molecules, such as CCL2, CX3CL1, EphA2, Slit, etc.; and (iii) neurotransmitters, including Ach, glutamate, glycine, epinephrine, norepinephrine, dopamine, etc. ${ }^{8,59}$. Unsurprisingly, tumor cells express various receptors, such as tyrosine kinase receptor A (TrkA), TrkB, and NGF receptor (NGFR), when responding to different neuroactive molecules to activate downstream pathways. Decades of research revealed the neuroactive molecules and receptors associated with tumor progression ${ }^{22,24,60-63}$. This way of communication between tumor cells and nerves is shown in Fig. 2.

In the prostate cancer model ${ }^{6}$, adrenergic fibers play a significant role through $\beta 2$ - and $\beta 3$-adrenergic receptors, whereas the cholinergic fibers act through the cholinergic receptors. The fact that $\beta$-blocker use can prolong survival in high-risk or metastatic prostate cancer patients is consistent with these findings ${ }^{64}$. Similar effects were also observed in skin cancer and breast cancer patients taking adrenergic antagonists ${ }^{65-67}$. Subsequently, Hayakawa et al. ${ }^{52}$ discovered that cholinergic stimulation by nerves through the release of Ach induced NGF expression in the gastric epithelium. In turn, NGF overexpression promoted cancer progression $^{52}$. Using the The Cancer Genome Atlas data, Deborde et al. ${ }^{68}$ revealed that a total of $48 \%$ of pancreatic cancer patients showed an alteration in genes coding neuroactive molecules or their receptors. The alternation of these genes was similar at $48 \%$ of patients with neuroendocrine prostate cancer and at $67 \%$ of patients with breast cancer. The gene whose expression changed most obviously was NTRK1 coding TrkA ${ }^{24}$. A further study demonstrated that TrkA expression could be found in $1.6 \%$ of solid tumors and was paralleled by the number of NTRK1 gene copies ${ }^{69}$. Inhibitors of TrkA have already shown a potential to treat NTRK fusion-positive cancer ${ }^{70}$.

The neurotransmitter ACh acts as an autocrine growth factor in human lung cancer and pancreatic cancer. Song et al. showed that ACh stimulated the proliferation of lung cancer cells via activating the mitogen-activated protein kinase and AKT pathways ${ }^{71,72}$. Apart from proliferation, ACh potently stimulates the adhesion, 


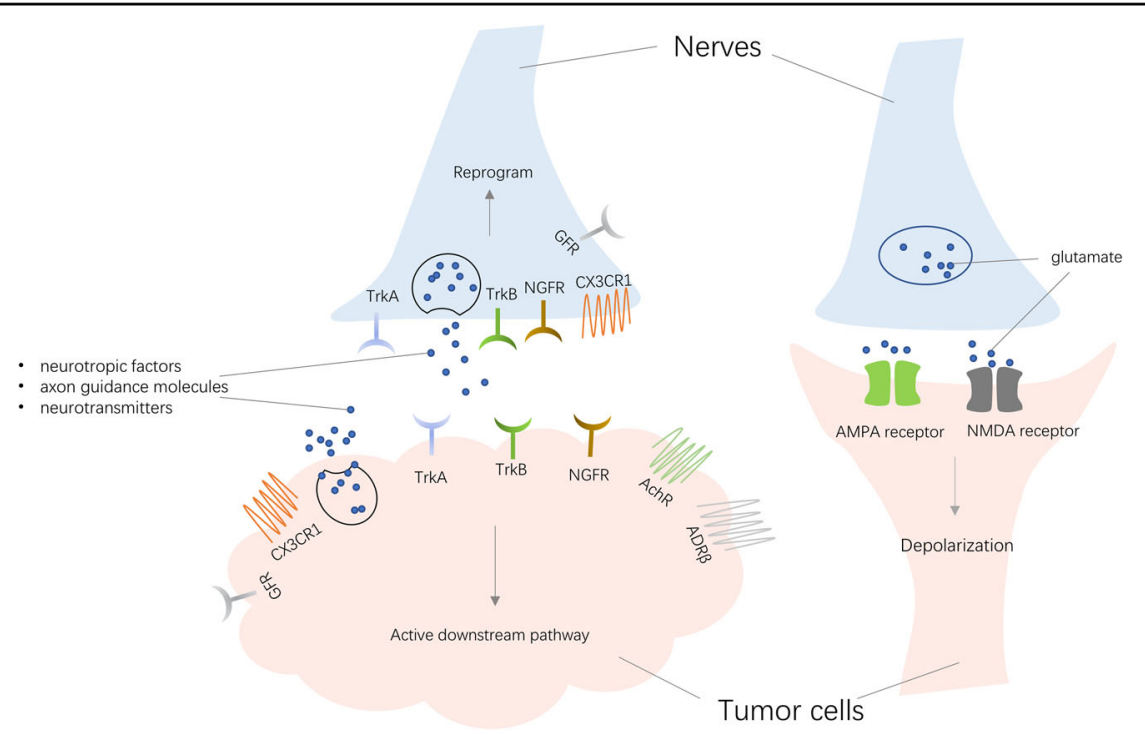

Fig. 2 Two direct ways of communication between tumor cells and nerves. Tumors release molecules also secreted by nerves, including neurotropic factors, axon guidance molecules, and neurotransmitters. These factors act on receptors of nerves to drive the nerve reprogramming, while they can also act on tumor receptors to activate downstream signaling. In addition to the paracrine model, tumors and nerves can form the synapses for depolarization to promote tumor progression. TrkA tyrosine kinase receptor A, TrkB tyrosine kinase receptor B, NGFR nerve growth factor receptor, AchR acetylcholine receptor, ADRB adrenoceptor $\beta$ receptor, CX3CR1 C-X3-C motif chemokine receptor 1, GFR glial cell-derived neurotrophic factor family receptor.

migration, and invasion of human lung cancer cells. Lin et al. ${ }^{73}$ observed that $\mathrm{ACh}$ increased the expression of MMP9 and downregulated the expression of E-cadherin. Both of these signaling events were associated with the migration and invasion phenotype in lung cancer. In another study, the stimulation of 7-nAChR enhanced pancreatic cancer metastasis via activating the JAK2/ STAT3 signaling cascade and the Ras/Raf/MEK/ERK1/2 pathway $^{74}$.

The above findings revealed that neuroactive molecules and receptors both participate in the tumor progression through the paracrine mode.

\section{Chemical synapse}

A special form of crosstalk between tumors and nerves is the chemical synapse, which is a structure that usually involves two adjacent neurons communicating using neurotransmitters, such as glutamate ${ }^{75}$. Synaptic structures involving presynaptic neurons and postsynaptic tumor cells in glioma were also observed by electron microscopy ${ }^{76,77}$. More importantly, by recording the excitatory postsynaptic potentials in glioma cells, both Venkatesh et al. ${ }^{76}$ and Venkataramani et al. ${ }^{77}$ indicated that these neurogliomal synapses may be functional in a similar manner to those formed between neurons. Gene expression analysis and confocal microscopy revealed that AMPA receptor was common in the postsynaptic region of glioma cells. Further studies on this receptor indicated that it mediated the depolarization of a glioma cell, which then spread through the network of glioma cells through their gap junctions. Crucially, neuronal activity or depolarization could promote tumor proliferation and invasion while preventing depolarization induced by synaptic activity, thus leading to a smaller tumor burden and longer survival time of animals ${ }^{78-80}$.

Zeng et al. ${ }^{81}$ also revealed that breast-to-brain metastasis (B2BM) cells establish pseudo-tripartite synapses between two neurons through the expression of neuroligin, which aids cell adhesion similar to the glioma ${ }^{81-83}$. High levels of the NMDA receptor (NMDAR) - in particular the subunit GLuN2B-was identified in B2BM cells through transcriptomic data. Furthermore, the currents and calcium transients after NMDAR activation were recorded. Cells produced smaller brain tumors and the mice had longer survival times after knocking down GLuN2B, suggesting that NMDAR synapses may promote the growth of cancer cells in the brain.

Taken together, these results demonstrate that nerves communicate with the tumors through establishing functional synapses to boost tumor progression. However, these tumors all occur in the brain environment and it remains unknown whether other solid tumors form synapses with nerves.

\section{Indirect effects of the nerves on tumor}

Nerves also interact with multiple stromal components in the TME to indirectly promote tumor growth and metastasis. Previous studies have proved that nerves 


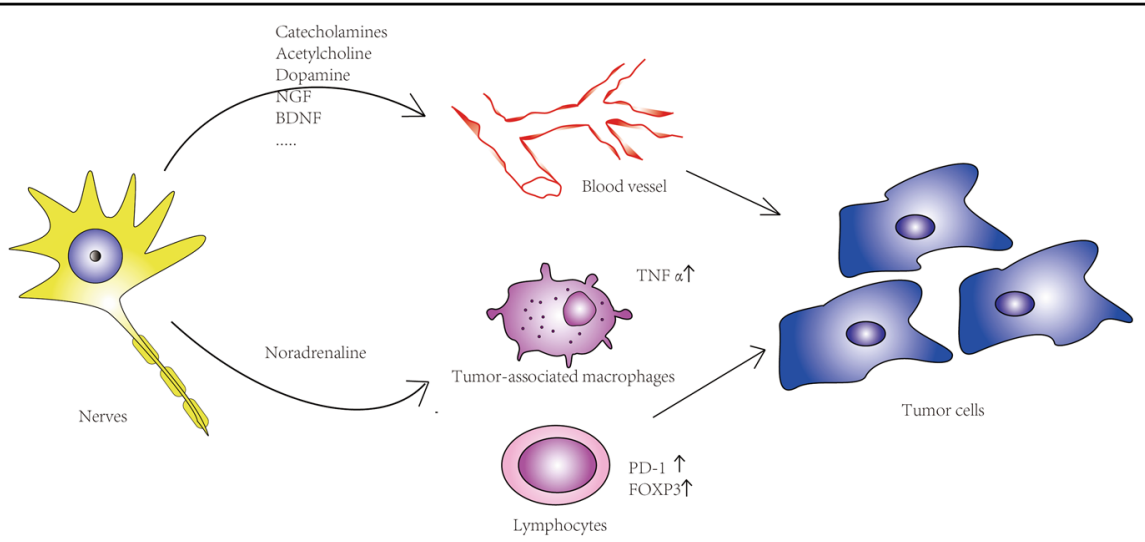

Fig. 3 Nerves affect tumor cell behaviors through indirect pathways. Nerves induce angiogenesis through secreting transmitters and neurotrophic factors. On the other hand, they regulate tumor-associated macrophages via cholinergic and adrenergic signaling. In addition, nerves mediate the expression of immune checkpoint molecules (PD-1, PD-L1 FOXP3) by lymphocytes; all the above processes influence tumor behaviors.

directly regulate stromal structures ${ }^{84}$. In this section, interactions between nerves and stromal compartments are discussed (Fig. 3).

\section{Angiogenesis}

The process of angiogenesis, which is the growth of new capillary vessels from existing vasculature by the activation, proliferation, and migration of endothelial cells, plays a crucial role in tumor growth and metastasis ${ }^{85-88}$. It allows tumors to develop their own nutrients and oxygen supply, thus enabling cell proliferation and tumor growth. Angiogenesis reflects the aggressiveness of tumor cells and associated with tumor outcomes ${ }^{89,90}$.

Transmitters and neurotrophic factors secreted by the nerves are involved in the process of angiogenesis through binding to receptors and inducing endothelial cells migration ${ }^{59}$. These factors, including catecholamines, Ach, dopamine, NGF, BNDF, etc., have been well summarized by Kuol and colleagues ${ }^{59,91-95}$. Recently, Zahalka et al. ${ }^{17}$ revealed that adrenergic nerves regulated angiogenesis in the prostate cancer microenvironment by altering the metabolism of blood vessel endothelial cells. Mechanistically, ADRB2 inhibited endothelial oxidative phosphorylation, which led to angiogenesis. The metabolic shift induced by nerves promoted prostate tumor growth through angiogenesis ${ }^{17}$.

Angiogenesis and neoneurogenesis indeed share a number of similarities. Both processes are regulated by similar transmitters and neurotrophic factors, and even share the same receptors ${ }^{96}$. All the above findings demonstrate that the regulation of angiogenesis and neoneurogenesis are closely intertwined.

\section{Immunity}

Nerves, as components of the TME, are also in crosstalk with the immune system, which could contribute to the tumor progression via inflammation ${ }^{97}$. These complex systems interact at multiple levels. Neuroendocrine and neuronal pathways are involved in the control of immune responses, as most of their molecular signals and receptors come from the same superfamily. In the spleen, e.g., adrenergic innervation was found to stimulate ACh production in $\beta 2$-adrenergic receptor ( $\beta 2-\mathrm{AR})$-expressing $\mathrm{T}$ cells ${ }^{98}$. T-cell-derived ACh has been recently reported to play an important role in regulating immunity, including cancer immunity. T-cell-derived ACh can inhibit tumor necrosis factor (TNF) production through the $\alpha 7$ nicotinic Ach receptor expressed by cytokineproducing macrophages ${ }^{99}$. The released $\mathrm{ACh}$ also binds back to nicotinic and muscarinic receptors on lung cancer cells to accelerate their proliferation, migration, and invasion ${ }^{100}$. Choline acetyltransferase, which catalyzes the synthesis of ACh from choline, is indeed strongly induced in both CD4+ and CD8+ T cells via IL-21, to regulate Tcell migration and immune functions ${ }^{101}$ (Fig. 4). In conclusion, these studies showed that the autonomic nervous system can directly regulate the immune system.

Tumor lymphocyte infiltration and activation are the important processes to inhibit tumor growth and progression $^{102}$. However, tumor cells escape immunosurveillance through the activation of immune checkpoint pathways that suppress antitumor immune responses ${ }^{103,104}$. A retrospective analysis of breast cancer patient revealed that sympathetic and parasympathetic nerve density correlated with the expression of immune checkpoint molecules (PD1, PD-L1, and FOXP3) and clinical outcomes ${ }^{4}$. This effect was also observed in animal experiments. Genetic sympathetic nerve denervation and parasympathetic neurostimulation reduced the expression of immune checkpoint molecules in a tumor tissue-specific and fiber type-specific manner in animal breast cancer models. These findings partly explained the opposing effects of sympathetic and 


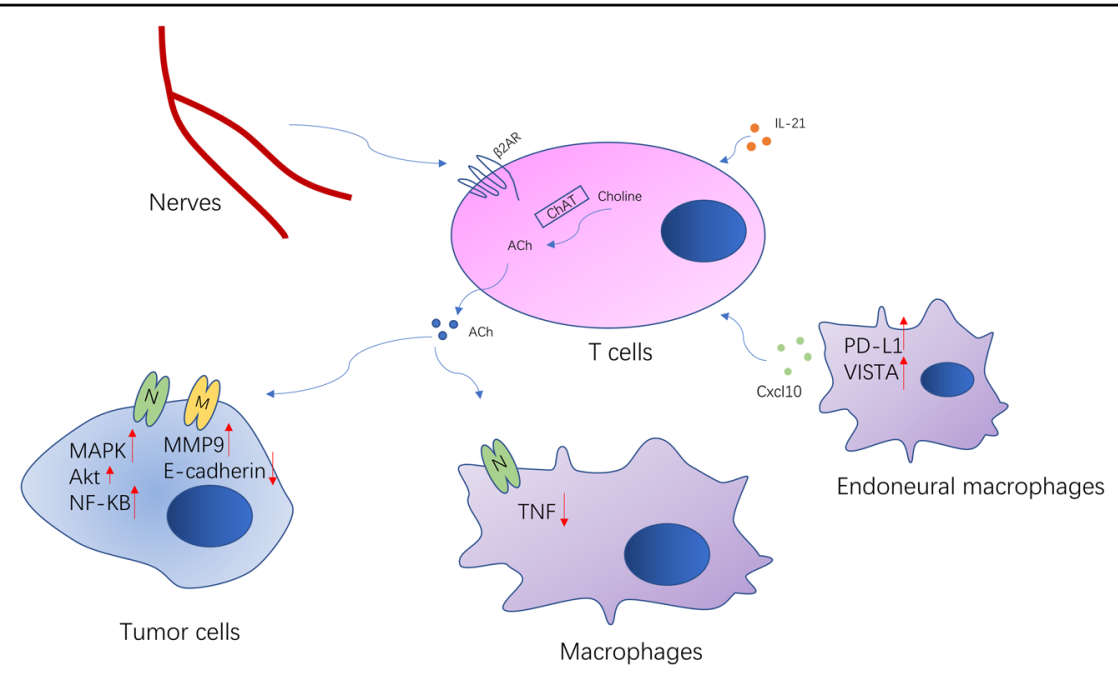

Fig. 4 Crosstalk between nerves and immune cells. ACh is synthesized in the T cells by enzyme choline acetyltransferase. Adrenergic innervation stimulates production of the T-cell-derived $\mathrm{ACh}$, which is involved in regulating the tumor immunity and tumor biology behavior. Endoneural macrophages also regulate tumor metastasis through CXCL10. CXCL10 recruits the myeloid cells and suppresses T cells via VISTA and PD-L1.

parasympathetic nerves in breast cancer, and indicated that nerves have a close association with immune checkpoint therapy. Nevertheless, the mechanism of how nerves regulate immune checkpoint molecules requires further elucidation.

TAMs, as essential components of the cancer microenvironment, play critical roles in the regulation of tumor development and progression ${ }^{105}$. TAMs can also modify the ability of tumor cells to resist cytotoxic chemotherapy via mediating the $\mathrm{TME}^{106}$. Interestingly, TAM recruitment is also regulated by both cholinergic and adrenergic signaling, which are related to the nerves. In pancreatic cancer, adrenergic signaling promotes tumor growth and reduces survival via TAM recruitment, while cholinergic signaling has the opposite effects ${ }^{54,107}$. A further study revealed that vagotomy promoted pancreatic cancer growth and reduced survival time through mediating TNFa secterion by $\mathrm{TAMs}^{53}$. Similar results were observed in the breast can$\operatorname{cer}^{108}$. Stress-induced neuroendocrine activation induced breast cancer metastasis to distant tissues, including the lung and lymph nodes. The pharmacological activation of $\beta$-adrenergic signaling induced similar effects, whereas a $\beta$-antagonist reversed these effects. Specifically, adrenergic signaling increased the infiltration of $\mathrm{CD} 11 \mathrm{~b}+\mathrm{F} 4 / 80+$ macrophages into the primary tumor and thereby induced a metastatic gene expression signature, accompanied by M2 macrophage differentiation.

Endoneural macrophages also participate in tumor metastasis. Microglia, as a type of native macrophages of the nervous system, are key promoters of brain metastasis ${ }^{109}$. Guldner et al. demonstrated that endoneural macrophages in the central nervous system drive immune suppression in the brain metastases through
CXCL10. Furthermore, macrophages could suppress Tcell activation to promote the brain metastases via VISTA and PD-L1 ${ }^{110}$. The elimination or inhibition of microglia function resulted in good antitumor metastasis effect. The blocking of any of the CCL2, STAT3, CSF-1R, and PI3K pathways of macrophages could inhibit brain metastasis ${ }^{111-114}$. However, more detailed investigations are needed to clarify the role of endoneural macrophages in tumorigenesis.

In summary, results suggest that nerves can regulate tumor progression through affecting the immune cells.

\section{Neural regulation in treatment resistance}

Owing to the deeper understanding of the underlying biological processes and molecular mechanisms of cancer progression, great progress has been made in cancer treatment. However, sooner or later, resistance develops to all kinds of therapy. Recent studies suggest that nerves and neural signals manipulate cancer therapeutic resistance ${ }^{115,116}$. This section aims to discuss the association between neural regulation and treatment resistance.

Chemotherapy is one of the most important applied therapeutic strategies for most cancers. Response to chemotherapeutics, however, varies greatly between individuals. Accumulating evidences suggest that the aberrant activation of adrenergic signaling affect sensitivity to cytotoxic chemotherapeutics by modulating the expression of other anti-apoptotic genes and inhibiting cellular apoptosis. Eng et al. ${ }^{117}$ reported that the activation of $\beta 2$-ARs resulted in changes in the apoptotic pathway regulation, which led to reduced therapeutic response. In cervical cancer cells, $\beta 2$-AR activation also induced chemoresistance by modulating 
p53 acetylation through upregulating SIRT1 $1^{118}$. A further study also indicated that $\beta 2$-AR remarkably impaired the chemotherapy response via upregulating DUSP $1^{119}$. These findings suggest that poor response to chemotherapeutics may be partly attributed to the abnormal functional activities of adrenergic signals.

Growing evidence reveals that nerves also stimulate a wide variety of signaling pathways causing resistance to drug therapy. Targeting members of the Epidermal Growth Factor Receptor family is an effective strategy for treating various cancers. Trastuzumab is the first line of therapy for Her2-positive breast cancer and gastric cancer $^{120,121}$. However, Trastuzumab resistance is a major clinical problem in the treatment of cancers ${ }^{122}$. Further evidence has suggested that $\beta 2-\mathrm{AR}$ is involved in the mechanism of Trastuzumab resistance. Shi et al. revealed that $\beta 2$-AR expression was positively correlated with Her2 expression in breast cancer; $\beta 2$-AR and Her2 comprised a positive feedback loop, where Her2 induced the upregulation of $\beta 2-A R$ via ERK pathway, whereas $\beta 2-A R$ induced the upregulation of $\mathrm{Her} 2^{123}$. Moreover, $\beta 2$-AR resulted in Trastuzumab resistance through mediating the PI3K/ AKT/mTOR pathway. Retrospective studies have also demonstrated that combining $\beta$-blockers with trastuzumab significantly improved survival in the patients with metastatic breast cancer ${ }^{124}$. It has also been reported that the activation of the $\beta 2$-AR signaling confers resistance to the tyrosine kinase inhibitor in the non-small cell lung cancer and hepatocellular carcinoma ${ }^{125,126}$.

Taken together, these results revealed that neural regulation was involved in tumor treatment resistance and targeting neural signaling pathway might be a potential strategy for treatment resistance.

\section{Conclusions}

In this review, it was highlighted that tumors can affect the nerves, which in turn, may modulate tumor biology via direct or indirect pathways. The detailed mode of tumor-nerve interaction was presented in Fig. 1. All of the listed evidences indicate that the nervous system is not a bystander with regards to cancer development and progression. Furthermore, some lines of evidences have linked the nerves to the treatment resistance. Due to the intimate relationship between nerves and tumor behavior, targeting nerves may provide novel strategies for the treatment of highly innervated cancers. As a result of nervous regulation of tumor angiogenesis and immunity, nerve targeting strategies could also be combined with anti-vascular therapy or immune therapy for a better cancer treatment effect. In recent years, the nerve targeting approach has already been applied in some clinical trials, but relevant methods are still far from clinical application.

For instance, surgical denervation significantly reduced gastric tumor incidence and progression ${ }^{50}$. However, the assessment of benefits and side effects of the procedure needs further investigation. The pharmacological inhibition of neural signaling is a promising target in anticancer therapy. Adrenergic signaling plays a critical role in tumor progression; thus, cancer treatment using $\beta$-adrenergic blockers remains controversial. Several lines of evidence have suggested that $\beta$-adrenergic blockers could prevent or reduce the mortality of various cancers, such as those of the pancreas, breast, and prostate ${ }^{127,128}$. Meanwhile, Heitz et al. claimed that selective $\beta$-blockers intake did not influence the prognosis for ovarian cancer patients. ${ }^{129}$ Regarding neurotrophic factors, NGF, BDNF, and their TrK receptors are the current research hotspots. However, the small-molecular targeting of TrKs has not been shown to have an impact on patient survival in clinical trials ${ }^{130,131}$; they even exhibit certain side effects by affecting other tyrosine kinases ${ }^{132}$. As a whole, further research is needed to identify the mechanisms of targeting the nerve pathway more specifically and to identify cancer patients that would benefit denervation procedures.

\section{Author contributions}

Y.C., G.L., and L.X. performed study concept and design. H.W. and Q.Z. performed development of methodology and writing, review, and revision of the paper. Z.L., L.W., and L.D. provided acquisition and analysis. H.Z. and M.W. provided technical and material support in revision. All authors read and approved the final paper.

\section{Funding}

This work was supported by Joint construction project of Zhejiang Province and Ministry (grant number: 2020388200); Key R\&D plan of Zhejiang Province (grant number: 2019C03089); National Natural Science Foundation of China (grant numbers: 81672520, 81870484, 81773789, and 81972367); Zhejiang Provincial Natural Science Foundation of China (grant numbers: LY17H160020); Zhejiang Medical and Health Science Plan Project (grant number: 2019ZD007 and 2019KY413).

Conflict of interest

The authors declare no competing interests.

\section{Publisher's note}

Springer Nature remains neutral with regard to jurisdictional claims in published maps and institutional affiliations.

Received: 14 December 2020 Revised: 8 February 2021 Accepted: 8 March 2021

Published online: 12 April 2021

\footnotetext{
References

1. Chen, F. et al. New horizons in tumor microenvironment biology: challenges and opportunities. BMC Med. 13, 45 (2015).

2. Wang, Y., Wang, L., Chen, C. \& Chu, X. New insights into the regulatory role of microRNA in tumor angiogenesis and clinical implications. Mol. Cancer 17, 22 (2018).

3. Saloman, J. L. et al. Ablation of sensory neurons in a genetic model of pancreatic ductal adenocarcinoma slows initiation and progression of cancer. Proc. Natl Acad. Sci. USA 113, 3078-3083 (2016).

4. Kamiya, A. et al. Genetic manipulation of autonomic nerve fiber innervation and activity and its effect on breast cancer progression. Nat. Neurosci. 22, 1289-1305 (2019).

5. Amit, M. et al. Loss of p53 drives neuron reprogramming in head and neck cancer. Nature 578, 449-454 (2020).
} 
6. Magnon, C. et al. Autonomic nerve development contributes to prostate cancer progression. Science 341, 1236361 (2013).

7. Tan, $X$. et al. Nerve fibers in the tumor microenvironment in neurotropic cancer-pancreatic cancer and cholangiocarcinoma. Oncogene 40, 899-908 (2020).

8. Saloman, J. L., Albers, K. M., Rhim, A. D. \& Davis, B. M. Can stopping nerves, stop cancer? Trends Neurosci. 39, 880-889 (2016).

9. Liebig, C. et al. Perineural invasion is an independent predictor of outcome in colorectal cancer. J. Clin. Oncol. 27, 5131-5137 (2009).

10. Huang, D. et al. Nerve fibers in breast cancer tissues indicate aggressive tumor progression. Medicine 93, e172 (2014).

11. Hibi, T. et al. Synuclein-gamma is closely involved in perineural invasion and distant metastasis in mouse models and is a novel prognostic factor in pancreatic cancer. Clin. Cancer Res. 15, 2864-2871 (2009).

12. Horn, L. C., Meinel, A., Fischer, U., Bilek, K. \& Hentschel, B. Perineural invasion in carcinoma of the cervix uteri-prognostic impact. J. Cancer Res. Clin. Oncol. 136, 1557-1562 (2010).

13. Tianhang, L., Guoen, F., Jianwei, B. \& Liye, M. The effect of perineural invasion on overall survival in patients with gastric carcinoma. J. Gastrointest. Surg. 12, 1263-1267 (2008).

14. Chen, J. W. et al. The prognostic effect of perineural invasion in esophageal squamous cell carcinoma. BMC Cancer 14, 313 (2014).

15. Chatterjee, D. et al. Perineural and intraneural invasion in posttherapy pancreaticoduodenectomy specimens predicts poor prognosis in patients with pancreatic ductal adenocarcinoma. Am. J. Surg. Pathol. 36, 409-417 (2012)

16. Ayala, G. E. et al. Cancer-related axonogenesis and neurogenesis in prostate cancer. Clin. Cancer Res. 14, 7593-7603 (2008).

17. Zahalka, A. H. et al. Adrenergic nerves activate an angio-metabolic switch in prostate cancer. Science 358, 321-326 (2017).

18. Horn, A., Dahl, O. \& Morild, I. Venous and neural invasion as predictors of recurrence in rectal adenocarcinoma. Dis. Colon Rectum 34, 798-804 (1991).

19. Batsakis, J. G. Nerves and neurotropic carcinomas. Ann. Otol. Rhinol. Laryngol. 94, 426-427 (1985)

20. Liebig, C., Ayala, G., Wilks, J. A., Berger, D. H. \& Albo, D. Perineural invasion in cancer: a review of the literature. Cancer 115, 3379-3391 (2009).

21. Bapat, A. A., Hostetter, G., Von Hoff, D. D. \& Han, H. Perineural invasion and associated pain in pancreatic cancer. Nat. Rev. Cancer 11, 695-707 (2011).

22. Arese, M., Bussolino, F., Pergolizzi, M., Bizzozero, L. \& Pascal, D. Tumor progression: the neuronal input. Ann. Transl. Med. 6, 89 (2018).

23. Demir, I. E. et al. Neural invasion in pancreatic cancer: the past, present and future. Cancers 2, 1513-1527 (2010)

24. Deborde, S. \& Wong, R. J. How Schwann cells facilitate cancer progression in nerves. Cell. Mol. life Sci. 74, 4405-4420 (2017).

25. Mavros, M. N., Economopoulos, K. P., Alexiou, V. G. \& Pawlik, T. M. Treatment and prognosis for patients with intrahepatic cholangiocarcinoma: systematic review and meta-analysis. JAMA Surg. 149, 565-574 (2014).

26. Hirai, I. et al. Perineural invasion in pancreatic cancer. Pancreas 24, 15-25 (2002).

27. Duraker, N., Sişman, S. \& Can, G. The significance of perineural invasion as a prognostic factor in patients with gastric carcinoma. Surg. today $\mathbf{3 3}, 95-100$ (2003).

28. He, P. et al. Multivariate statistical analysis of clinicopathologic factors influencing survival of patients with bile duct carcinoma. World J. Gastroenterol. $\mathbf{8}$ 943-946 (2002).

29. Lee, I. H. et al. Perineural invasion is a marker for pathologically advanced disease in localized prostate cancer. Int. J. Radiat. Oncol. Biol. Phys. 68, 1059-1064 (2007).

30. Schmitd, L. B., Scanlon, C. S. \& D'Silva, N. J. Perineural invasion in head and neck cancer. J. Dent. Res. 97, 742-750 (2018).

31. Amit, M., Na'ara, S. \& Gil, Z. Mechanisms of cancer dissemination along nerves. Nat. Rev. Cancer 16, 399-408 (2016).

32. De Oliveira, T. et al. Syndecan-2 promotes perineural invasion and cooperates with K-ras to induce an invasive pancreatic cancer cell phenotype. Mol. Cancer 11, 19 (2012)

33. Marchesi, F. et al. The chemokine receptor CX3CR1 is involved in the neural tropism and malignant behavior of pancreatic ductal adenocarcinoma. Cancer Res. 68, 9060-9069 (2008).

34. Abiatari, l. et al. Consensus transcriptome signature of perineural invasion in pancreatic carcinoma. Mol. Cancer Ther. 8, 1494-1504 (2009).
35. Li, X. et al. Sonic hedgehog paracrine signaling activates stromal cells to promote perineural invasion in pancreatic cancer. Clin. Cancer Res. 20 4326-4338 (2014).

36. Demir, I. E. et al. Perineural mast cells are specifically enriched in pancreatic neuritis and neuropathic pain in pancreatic cancer and chronic pancreatitis. PLOS ONE 8, e60529 (2013).

37. Cavel, O. et al. Endoneurial macrophages induce perineural invasion of pancreatic cancer cells by secretion of GDNF and activation of RET tyrosine kinase receptor. Cancer Res. 72, 5733-5743 (2012).

38. Makwana, M. \& Raivich, G. Molecular mechanisms in successful peripheral regeneration. FEBS J. 272, 2628-2638 (2005).

39. Idiaquez J., Benarroch E. \& Nogues M. In Evaluation and Management of Autonomic Disorders: A Case-Based Practical Guide (eds. Idiaquez J., Benarroch E. \& Nogues M.) 3-18 (Springer, 2018).

40. McCorry, L. K. Physiology of the autonomic nervous system. Am. J. Pharm. Educ. 71, 78 (2007).

41. McVary, K. T. et al. Growth of the rat prostate gland is facilitated by the autonomic nervous system. Biol. Reprod. 51, 99-107 (1994).

42. McVary, K. T., McKenna, K. E. \& Lee, C. Prostate innervation. Prostate Suppl. 8 2-13 (1998)

43. Raju, B., Haug, S. R., Ibrahim, S. O. \& Heyeraas, K. J. Sympathectomy decreases size and invasiveness of tongue cancer in rats. Neuroscience 149, 715-725 (2007)

44. Kappos, E. A. et al. Denervation leads to volume regression in breast cancer. J. Plast. Reconstruct. Aesth. Surg. 71, 833-839 (2018).

45. Schwartz, E. S. et al. Synergistic role of TRPV1 and TRPA1 in pancreatic pain and inflammation. Gastroenterology 140, 1283-1291 (2011).

46. Schwartz, E. S. et al. TRPA1 and TRPA1 antagonists prevent the transition of acute to chronic inflammation and pain in chronic pancreatitis. J. Neurosci. 33, 5603-5611 (2013).

47. Stopczynski, R. E. et al. Neuroplastic changes occur early in the development of pancreatic ductal adenocarcinoma. Cancer Res. 74, 1718-1727 (2014).

48. Peterson, S. C. et al. Basal cell carcinoma preferentially arises from stem cells within hair follicle and mechanosensory niches. Cell Stem Cell 16, 400-412 (2015).

49. Berthoud, H. R. \& Neuhuber, W. L. Functional and chemical anatomy of the afferent vagal system. Auton. Neurosci. 85, 1-17 (2000).

50. Zhao, C. M. et al. Denervation suppresses gastric tumorigenesis. Sci. Transl. Med. 6, 250ra115 (2014).

51. Polli-Lopes, A. C., Zucoloto, S., de Queirós Cunha, F., da Silva Figueiredo, L. A. \& Garcia, S. B. Myenteric denervation reduces the incidence of gastric tumors in rats. Cancer Lett. 190, 45-50 (2003).

52. Hayakawa, $Y$. et al. Nerve growth factor promotes gastric tumorigenesis through aberrant cholinergic signaling. Cancer Cell 31, 21-34 (2017).

53. Partecke, L. I. et al. Subdiaphragmatic vagotomy promotes tumor growth and reduces survival via TNFalpha in a murine pancreatic cancer model. Oncotarget 8, 22501-22512 (2017).

54. Renz, B. W. et al. Cholinergic signaling via muscarinic receptors directly and indirectly suppresses pancreatic tumorigenesis and cancer stemness. Cancer Discov. 8, 1458-1473 (2018).

55. Zhu, Y. et al. Tissue-resident macrophages in pancreatic ductal adenocarcinoma originate from embryonic hematopoiesis and promote tumor progression. Immunity 47, 323-338.e326 (2017).

56. Partecke, L. I. et al. Subdiaphragmatic vagotomy promotes tumor growth and reduces survival via TNFa in a murine pancreatic cancer model. Oncotarget 8, 22501-22512 (2017).

57. Mauffrey, P. et al. Progenitors from the central nervous system drive neurogenesis in cancer. Nature 569, 672-678 (2019).

58. Scuteri, A. et al. Mesenchymal stem cells neuronal differentiation ability: a real perspective for nervous system repair? Curr. Stem Cell Res. Ther. 6, 82-92 (2011).

59. Kuol, N., Stojanovska, L., Apostolopoulos, V. \& Nurgali, K. Role of the nervous system in tumor angiogenesis. Cancer Microenviron. 11, 1-11 (2018).

60. Mancino, M., Ametller, E., Gascón, P. \& Almendro, V. The neuronal influence on tumor progression. Biochim. Biophys. Acta 1816, 105-118 (2011).

61. Barquilla, A. \& Pasquale, E. B. Eph receptors and ephrins: therapeutic opportunities. Annu. Rev. Pharmacol. Toxicol. 55, 465-487 (2015).

62. Rehman, M. \& Tamagnone, L. Semaphorins in cancer: biological mechanisms and therapeutic approaches. Semin. Cell Dev. Biol. 24, 179-189 (2013). 
63. Jiang, S. H., Hu, L. P., Wang, X., Li, J. \& Zhang, Z. G. Neurotransmitters: emerging targets in cancer. Oncogene 39, 503-515 (2020).

64. Grytli, H. H., Fagerland, M. W., Fosså, S. D. \& Taskén, K. A. Association between use of $\beta$-blockers and prostate cancer-specific survival: a cohort study of 3561 prostate cancer patients with high-risk or metastatic disease. Eur. Urol. 65, 635-641 (2014)

65. Yang, E. V. \& Eubank, T. D. The impact of adrenergic signaling in skin cancer progression: possible repurposing of $\beta$-blockers for treatment of skin cancer. Cancer Biomark. 13, 155-160 (2013).

66. Phadke, S. \& Clamon, G. Beta blockade as adjunctive breast cancer therapy: a review. Crit. Rev. Oncol. Hematol. 138, 173-177 (2019).

67. Spini, A. et al. Evidence of $\beta$-blockers drug repurposing for the treatment of triple negative breast cancer: a systematic review. Neoplasma 66, 963-970 (2019).

68. Witkiewicz, A. K. et al. Whole-exome sequencing of pancreatic cancer defines genetic diversity and therapeutic targets. Nat. Commun. 6, 6744 (2015).

69. Mauri, G. et al. TRKA expression and NTRK1 gene copy number across solid tumours. J. Clin. Pathol. 71, 926-931 (2018).

70. Cocco, E., Scaltriti, M. \& Drilon, A. NTRK fusion-positive cancers and TRK inhibitor therapy. Nat. Rev. Clin. Oncol. 15, 731-747 (2018).

71. Song, P. et al. Acetylcholine is synthesized by and acts as an autocrine growth factor for small cell lung carcinoma. Cancer Res. 63, 214-221 (2003).

72. Song, P. et al. Activated cholinergic signaling provides a target in squamous cell lung carcinoma. Cancer Res. 68, 4693-4700 (2008).

73. Lin, G., Sun, L., Wang, R., Guo, Y. \& Xie, C. Overexpression of muscarinic receptor 3 promotes metastasis and predicts poor prognosis in non-smallcell lung cancer. J. Thorac. Oncol. 9, 170-178 (2014).

74. Momi, N. et al. Nicotine/cigarette smoke promotes metastasis of pancreatic cancer through a7nAChR-mediated MUC4 upregulation. Oncogene 32 1384-1395 (2013).

75. Choquet, D. \& Triller, A. The dynamic synapse. Neuron 80, 691-703 (2013).

76. Venkatesh, H. S. et al. Electrical and synaptic integration of glioma into neural circuits. Nature 573, 539-545 (2019).

77. Venkataramani, V. et al. Glutamatergic synaptic input to glioma cells drives brain tumour progression. Nature 573, 532-538 (2019).

78. Rzeski, W., Turski, L. \& Ikonomidou, C. Glutamate antagonists limit tumor growth. Proc. Natl Acad. Sci. USA 98, 6372-6377 (2001).

79. Savaskan, N. E. et al. Small interfering RNA-mediated $x \subset T$ silencing in gliomas inhibits neurodegeneration and alleviates brain edema. Nat. Med. 14 629-632 (2008).

80. Takano, T. et al. Glutamate release promotes growth of malignant gliomas. Nat. Med. 7, 1010-1015 (2001).

81. Zeng, Q. et al. Synaptic proximity enables NMDAR signalling to promote brain metastasis. Nature 573, 526-531 (2019).

82. Gambrill, A. C. \& Barria, A. NMDA receptor subunit composition controls synaptogenesis and synapse stabilization. Proc. Natl Acad. Sci. USA 108, 5855-5860 (2011).

83. Li, L. \& Hanahan, D. Hijacking the neuronal NMDAR signaling circuit to promote tumor growth and invasion. Cell 153, 86-100 (2013).

84. Kepper, M. \& Keast, J. Immunohistochemical properties and spinal connections of pelvic autonomic neurons that innervate the rat prostate gland. Cell Tissue Res. 281, 533-542 (1995).

85. Folkman, J. Tumor angiogenesis: therapeutic implications. N. Engl. J. Med. 285, 1182-1186 (1971).

86. De Palma, M., Biziato, D. \& Petrova, T. V. Microenvironmental regulation of tumour angiogenesis. Nat. Rev. Cancer 17, 457-474 (2017).

87. Chung, H. C. Angiogenesis in cancer. Vasc. Health Risk Manag. 2, 213-219 (2001).

88. Folkman, J., Watson, K., Ingber, D. \& Hanahan, D. Induction of angiogenesis during the transition from hyperplasia to neoplasia. Nature 339, 58-61 (1989).

89. Li, S. et al. Angiogenesis in pancreatic cancer: current research status and clinical implications. Angiogenesis 22, 15-36 (2019).

90. Sharma, S., Sharma, M. C. \& Sarkar, C. Morphology of angiogenesis in human cancer: a conceptual overview, histoprognostic perspective and significance of neoangiogenesis. Histopathology 46, 481-489 (2005).

91. Chakroborty, D., Sarkar, C., Basu, B., Dasgupta, P. S. \& Basu, S. Catecholamines regulate tumor angiogenesis. Cancer Res. 69, 3727-3730 (2009).
92. Fiszman, G. L. et al. Activation of muscarinic cholinergic receptors induces MCF-7 cells proliferation and angiogenesis by stimulating nitric oxide synthase activity. Cancer Biol. Ther. 6, 1106-1113 (2007).

93. Chakroborty, D. et al. Depleted dopamine in gastric cancer tissues: dopamine treatment retards growth of gastric cancer by inhibiting angiogenesis. Clin Cancer Res. 10, 4349-4356 (2004).

94. Romon, R. et al. Nerve growth factor promotes breast cancer angiogenesis by activating multiple pathways. Mol. Cancer 9, 157 (2010).

95. Lin, C. Y. et al. Brain-derived neurotrophic factor increases vascular endothelial growth factor expression and enhances angiogenesis in human chondrosarcoma cells. Biochem. Pharmacol. 91, 522-533 (2014).

96. Eichmann, A. \& Brunet, I. Arterial innervation in development and disease. Sci. Transl. Med. 6, 252ps259 (2014)

97. Wrona, D. Neural-immune interactions: an integrative view of the bidirectional relationship between the brain and immune systems. J. Neuroimmunol. 172, 38-58 (2006)

98. Rosas-Ballina, M. et al. Acetylcholine-synthesizing T cells relay neural signals in a vagus nerve circuit. Science 334, 98-101 (2011).

99. Wang, $\mathrm{H}$. et al. Nicotinic acetylcholine receptor alpha7 subunit is an essential regulator of inflammation. Nature 421, 384-388 (2003).

100. Friedman, J. R. et al. Acetylcholine signaling system in progression of lung cancers. Pharmacol. Ther. 194, 222-254 (2019).

101. Cox, M. A. et al. Choline acetyltransferase-expressing $T$ cells are required to control chronic viral infection. Science 363, 639-644 (2019).

102. Salmon, H., Remark, R., Gnjatic, S. \& Merad, M. Host tissue determinants of tumour immunity. Nat. Rev. Cancer 19, 215-227 (2019).

103. Darvin, P., Toor, S. M., Sasidharan Nair, V. \& Elkord, E. Immune checkpoint inhibitors: recent progress and potential biomarkers. Exp. Mol. Med. 50, 1-11 (2018).

104. Sharma, P. \& Allison, J. P. The future of immune checkpoint therapy. Science 348, 56-61 (2015).

105. Partecke, L. I. et al. Induction of M2-macrophages by tumour cells and tumour growth promotion by M2-macrophages: a quid pro quo in pancreatic cancer. Pancreatology 13, 508-516 (2013).

106. Sanchez, L. R. et al. The emerging roles of macrophages in cancer metastasis and response to chemotherapy. J. Leukoc. Biol. 106, 259-274 (2019).

107. Partecke, L. I. et al. Chronic stress increases experimental pancreatic cancer growth, reduces survival and can be antagonised by beta-adrenergic receptor blockade. Pancreatology 16, 423-433 (2016).

108. Sloan, E. K. et al. The sympathetic nervous system induces a metastatic switch in primary breast cancer. Cancer Res. 70, 7042-7052 (2010).

109. He, B. P. et al. Differential reactions of microglia to brain metastasis of lung cancer. Mol. Med. 12, 161-170 (2006).

110. Guldner, I. H. et al. CNS-Native Myeloid Cells Drive Immune Suppression in the Brain Metastatic Niche through Cxcl10. Cell 183, 1234-1248.e25 (2020).

111. Qian, B. Z. et al. CCL2 recruits inflammatory monocytes to facilitate breasttumour metastasis. Nature 475, 222-225 (2011).

112. Pyonteck, S. M. et al. CSF-1R inhibition alters macrophage polarization and blocks glioma progression. Nat. Med. 19, 1264-1272 (2013).

113. Blazquez, R. et al. PI3K: a master regulator of brain metastasis-promoting macrophages/microglia. Glia 66, 2438-2455 (2018).

114. You, H., Baluszek, S. \& Kaminska, B. Immune microenvironment of brain metastases-are microglia and other brain macrophages little helpers? Front. Immunol. 10, 1941 (2019)

115. Liu, D. et al. Neural regulation of drug resistance in cancer treatment. Biochim Biophys. Acta Rev. Cancer 1871, 20-28 (2019).

116. Boshuizen, J. et al. Reversal of pre-existing NGFR-driven tumor and immune therapy resistance. Nat. Commun. 11, 3946 (2020).

117. Eng, J. W. et al. Housing temperature-induced stress drives therapeutic resistance in murine tumour models through $\beta 2$-adrenergic receptor activation. Nat. Commun. 6, 6426 (2015).

118. Chen, $\mathrm{H}$. et al. beta2-AR activation induces chemoresistance by modulating p53 acetylation through upregulating Sirt1 in cervical cancer cells. Cancer Sci. 108, 1310-1317 (2017)

119. Kang, Y. et al. Adrenergic stimulation of DUSP1 impairs chemotherapy response in ovarian cancer. Clin. Cancer Res. 22, 1713-1724 (2016).

120. Figueroa-Magalhães, M. C., Jelovac, D., Connolly, R. \& Wolff, A. C. Treatment of HER2-positive breast cancer. Breast 23, 128-136 (2014).

121. Smyth, E. C., Nilsson, M., Grabsch, H. I., van Grieken, N. C. \& Lordick, F. Gastric cancer. Lancet 396, 635-648 (2020). 
122. Madrid-Paredes, A., Cañadas-Garre, M., Sánchez-Pozo, A. \& Calleja-Hernández, M. Non-HER2 signaling pathways activated in resistance to anti-HER2 therapy in breast cancer. Breast Cancer Res. Treat. 153, 493-505 (2015).

123. Shi, $M$. et al. The $\beta 2$-adrenergic receptor and Her2 comprise a positive feedback loop in human breast cancer cells. Breast Cancer Res. Treat. 125, 351-362 (2011).

124. Liu, D. et al. $\beta 2-A R$ signaling controls trastuzumab resistance-dependent pathway. Oncogene 35, 47-58 (2016).

125. Nilsson, M. B. et al. Stress hormones promote EGFR inhibitor resistance in NSCLC: Implications for combinations with beta-blockers. Sci. Transl. Med. 9 eaao4307 (2017)

126. Wu, F. Q. et al. ADRB2 signaling promotes HCC progression and sorafenib resistance by inhibiting autophagic degradation of HIF1a. J. Hepatol. $\mathbf{6 5}$, 314-324 (2016).

127. Al-Wadei, H. A., Al-Wadei, M. H. \& Schuller, H. M. Prevention of pancreatic cancer by the beta-blocker propranolol. Anticancer Drugs 20,477-482 (2009).
128. Powe, D. G. et al. Beta-blocker drug therapy reduces secondary cancer formation in breast cancer and improves cancer specific survival. Oncotarget 1, 628-638 (2010).

129. Heitz, F. et al. Impact of beta blocker medication in patients with platinum sensitive recurrent ovarian cancer-a combined analysis of 2 prospective multicenter trials by the AGO Study Group, NCIC-CTG and EORTC-GCG. Gynecol. Oncol. 129, 463-466 (2013).

130. Smith, M. et al. Phase III study of cabozantinib in previously treated metastatic castration-resistant prostate cancer: COMET-1. J. Clin. Oncol. 34, 3005-3013 (2016).

131. Collins, C. et al. Preclinical and clinical studies with the multi-kinase inhibitor CEP-701 as treatment for prostate cancer demonstrate the inadequacy of PSA response as a primary endpoint. Cancer Biol. Ther. 6 , 1360-1367 (2007).

132. Shabbir, M. \& Stuart, R. Lestaurtinib, a multitargeted tyrosine kinase inhibitor: from bench to bedside. Expert Opin. Invest. Drugs 19, 427-436 (2010). 